\title{
Adsorption de protéines sur une électrode de dioxyde d'étain polarisée anodiquement en présence d'ions chlorure
}

\author{
S. Haskouri ${ }^{1}$, H. Cachet $^{1}$, C. Debiemme-Chouvy ${ }^{1}$, R. Warocquier-Clerout ${ }^{2}$ \\ et M.D. Nagel ${ }^{2}$ \\ ${ }^{1}$ CNRS-UPR 15, Laboratoire Interfaces et Systèmes Électrochimiques (LISE), Université P. et M. Curie, Paris, \\ France \\ e-mail : huc@ccr.jussieu.fr \\ 2 UMR 6600 CNRS, Université de Technologie de Compiègne, Biomécanique et Génie Médical, Compiègne, France
}

Mots-clés :

Dioxyde d'étain ; adsorption de protéines ; acide

hypochloreux ; biosalissures
Résumé - L'oxydation électrochimique des ions chlorure produit l'acide hypochloreux qui est un oxydant puissant. Cette réaction est utilisée pour protéger, vis-à-vis des biosalissures, des dispositifs optiques immergés en milieu marin, la réaction se déroulant sur une électrode transparente en dioxyde d'étain. Après une séquence sous polarisation, la fenêtre optique reste exempte de salissures pendant une à deux semaines. Nous avions précédemment recherché, sans succès, la formation éventuelle de composés inorganiques chlorés. Nous étudions actuellement la possibilité d'une contribution de la matière organique ayant interagi avec l'acide hypochloreux produit sous polarisation. Celui-ci peut en effet réagir avec des molécules biologiques comme les protéines et les modifier. Nous avons choisi ici comme protéine modèle l'albumine du sérum bovin (BSA). Son adsorption sur $\mathrm{SnO}_{2}$ a été étudiée in situ à l'aide d'une microbalance à quartz en fonction de sa concentration volumique, de la concentration en ions $\mathrm{Cl}^{-}$et du potentiel appliqué. Les milieux d'étude sont soit des solutions de $B S A$, soit du sérum de veau fœtal SVF, additionnés de $\mathrm{NaCl} 0,5 \mathrm{M}$ pour se rapprocher des conditions de l'eau de mer. Sous polarisation à $+1,5 \mathrm{~V} / \mathrm{ECS}$, la BSA s'adsorbe notablement, avec un bon accord entre les mesures in situ par microbalance et ex situ, par des tests biologiques et des analyses XPS. Ces dernières révèlent la présence de chlore dans le film organique, suggérant la formation de groupements chloramine qui pourraient constituer l'agent antisalissure.
Key words:

Tin dioxide; protein adsorption; hypochlorous acid; antifouling
Abstract - Protein adsorption at anodically polarised tin dioxide electrodes in chloride containing solutions. Hypochlorous acid, a powerful oxidising agent, is readily obtained by electrochemical oxidation of chloride ions. This reaction is used to protect underwater optical instrumentation against biofouling, seawater electrolysis taking place at a conductive and transparent tin dioxide coating. After an anodic polarization sequence, the window was seen to remain free of biofouling for one or two weeks. A purely inorganic origin of this effect was firstly under consideration (as hydroxochloride formation) but it had to be ruled out. Another way is to consider the interaction of organic matter with hypochlorous species electrochemically produced. In this work, bovine serum albumin (BSA) was taken as a protein model. Quartz crystal microbalance (QCM) was used to study BSA adhesion on $\mathrm{SnO}_{2}$ as a function of BSA, chloride bulk concentrations and applied potential. $\mathrm{NaCl}$ concentration was adjusted to $0.5 \mathrm{M}$ by adding $\mathrm{NaCl}$ to simulate natural seawater. At $+1.5 \mathrm{~V} / \mathrm{SCE}$, the working conditions for active antifouling protection, BSA is found to be strongly adsorbed on $\mathrm{SnO}_{2}$. A good agreement is obtained between in situ QCM measurements and ex situ biological tests and XPS analyses. The latter show that chlorine is present in the organic layer at the $\mathrm{SnO}_{2}$ surface, suggesting the formation of chloramine groups with likely antifouling properties. 
1 est bien établi que l'adsorption de protéines sur un substrat constitue une première étape dans la formation de biosalissures [1]. Ce phénomène a lieu sur toute surface immergée dans des eaux douces ou salées, tels que rochers, installations portuaires ou offshore, coques de navires, échangeurs de chaleur, instrumentations sous-marines. Il est également connu dans le domaine biomédical qui utilise des implants, des prothèses, des cathéters et dans l'industrie agro-alimentaire. L'adsorption d'une macromolécule est généralement considérée comme irréversible du fait de la multiplicité des sites actifs possibles. Elle met en jeu principalement des forces de Van der Waals, des forces électrostatiques et à très courtes distances, des interactions chimiques, par exemple de type acido-basique. De nombreux travaux ont été et sont consacrés à l'étude du processus d'adsorption de protéines via l'influence de divers paramètres agissant sur les interactions entre protéines et surface : nature du substrat, nature de la protéine, $\mathrm{pH}$, concentration en protéines et en ions, température, charges de surface $[1,2]$.

Dans le présent travail, nous nous intéressons à l'interaction d'une surface de dioxyde d'étain avec un milieu électrolytique contenant des ions chlorure et des protéines. L'origine de cette étude réside dans l'observation faite de propriétés antisalissures acquises en milieu marin par un film de $\mathrm{SnO}_{2}$ sur lequel a été produit électrochimiquement de l'acide hypochloreux $(\mathrm{HClO})$ [3]. Le contexte est celui de la mise en œuvre d'un procédé de protection électrochimique de hublots immergés en milieu marin sur de longues durées, en collaboration avec IFREMER-Centre de Brest [4]. Il requiert le dépôt à la surface du hublot en verre d'un film de $\mathrm{SnO}_{2}$ conducteur et transparent dont les caractéristiques physicochimiques ont été optimisées en vue de son fonctionnement en tant qu'anode, à un potentiel de $+1,5 \mathrm{~V} / \mathrm{ECS}$ pour produire $\mathrm{HClO}$ [5]. La description et les avantages du procédé, sa mise en œuvre sur différents sites ont été présentés antérieurement [3,4 et références incluses]. Sous polarisation anodique, l'action antisalissure repose sur la génération de chlore actif à la surface du hublot. Dans ce cas, le principe de la protection est clair. Ce qui ne l'est pas, c'est pourquoi une protection persiste sur une à deux semaines après coupure de la polarisation électrique. La compréhension du mécanisme d'action de ce mode de protection passif est un point important, car il permettrait d'envisager la préparation en laboratoire de surfaces modifiées ayant des propriétés antifouling et/ou d'optimiser le mode de protection actif (durée de maintien sous/hors polarisation).

Dans une première approche, nous avons recherché une origine purement inorganique à ce problème. En effet, comme nous l'avons montré, sous forte polarisation anodique, la surface de $\mathrm{SnO}_{2}$ subit l'action des espèces radicalaires $\mathrm{OH}^{\bullet}$ et $\mathrm{Cl}^{\bullet}$ électrogénérées conduisant à la formation de groupements Sn-OHetSn-Clen surface. La question posée était de savoir si une telle modification de la surface pouvait être responsable partiellement ou totalement de l'effet antibiofilm, sachant que des objets en étain recouverts d'hydroxochlorures d'étain, trouvés dans des épaves, sont dépourvus de biofilm [6]. La réponse étant négative [7], on considère maintenant la possibilité d'une contribution de la matière organique adsorbée et de son interaction avec les espèces chlorées produites sous polarisation anodique. L'albumine du sérum bovin (BSA) a été choisie comme protéine modèle $[1,2,8,9]$. Le point isoélectrique de la BSA correspond à un $\mathrm{pH}$ de 4,7 [10,11]. La protéine est donc chargée négativement à des $\mathrm{pH}$ voisins de la neutralité. À ces $\mathrm{pH}$, la charge de surface de $\mathrm{SnO}_{2}$, due à ses propriétés acide-base, est plutôt négative, ce qui a priori gênerait l'adsorption de la BSA pour des raisons de répulsions électrostatiques. Asanov et coll. ont étudié l'influence du potentiel entre - 0,6 et $+1,5 \mathrm{~V}(\mathrm{Ag} / \mathrm{AgCl})$ sur l'adsorption sur $\mathrm{SnO}_{2}$ de la BSA diluée dans un tampon phosphate à $\mathrm{pH} 7,4$ [2]. L'adsorption est faible aux potentiels positifs et augmente aux potentiels les plus négatifs, en accord avec une augmentation de l'hydrophobicité de la surface. Nous verrons qu'en présence d'ions chlorure les conclusions sont différentes. Par ailleurs, quelques travaux ont montré que l'oxydation de protéines par $\mathrm{HClO}$ entraîne la formation de groupements chloramine et de radicaux pouvant conduire à la fragmentation de la protéine [12-14]. De tels groupements chloramine portés par des protéines adsorbées à la surface de $\mathrm{SnO}_{2}$ pourraient être à l'origine de la protection antisalissure persistant après coupure de la polarisation.

Dans cette perspective, nous avons étudié l'adsorption de la BSA sur un film $\mathrm{SnO}_{2}$, en combinant des mesures de masse in situ par microbalance à quartz électrochimique (QCM) et des analyses ex situ par des tests biochimiques après désorption (SDSPAGE [15] et dosages protéiques) et par 
spectroscopie de photoélectrons induits par rayonnement $X(X P S)$. L'influence du potentiel appliqué a été étudiée à différentes concentrations en BSA $(0,2$ à $5 \%)$ et en $\mathrm{NaCl}$, 0,5 M étant la molarité retenue pour comparaison avec l'eau de mer.

\section{Détails expérimentaux}

Les films de dioxyde d'étain sont déposés par pyrolyse de spray sur des lames de verre pour microscopie ou sur des quartz piézoélectriques pour les mesures QCM (coupe AT - $6 \mathrm{MHz}$ - coefficient de sensibilité fréquence/masse : $-7,5 \times 10^{7} \mathrm{~Hz} \mathrm{~g}^{-1} \mathrm{~cm}^{2}$ [16]). Selon le procédé CNRS-IFREMER, les films $\mathrm{SnO}_{2}$ sont constitués d'une sous couche dopée au fluor, recouverte d'une couche dopée à l'antimoine, chaque couche ayant une épaisseur de $300 \mathrm{~nm}$ [3]. La résistance carré est comprise entre 10 et $15 \mathrm{ohms}$. Selon les expériences, nous avons solubilisé de la BSA (Sigma) (masse moléculaire: $66 \mathrm{kD}$, taille : $14 \times 4 \times 4 \mathrm{~nm}^{3}$ ) dans une solution aqueuse de $\mathrm{NaCl} 0,15 \mathrm{M}$ ou $0,5 \mathrm{M}$ à des concentrations variant de 0,2 à $5 \%$ ou nous avons choisi du sérum de veau fœetal SVF (Gibco) qui renferme différentes protéines dont l'albumine est majoritaire avec $30 \mathrm{mg} \mathrm{cm}^{-3}$ (3\%). On ajuste le cas échéant la concentration en $\mathrm{NaCl}$ à $0,5 \mathrm{M}$. Les mesures électrochimiques et électrogravimétriques ont été effectuées dans une cellule à trois électrodes de volume $20 \mathrm{~cm}^{3}$. Tous les potentiels sont mesurés par rapport à une électrode de référence au calomel en solution saturée de $\mathrm{KCl}$ (ECS). La composition chimique des surfaces (épaisseur $<10 \mathrm{~nm}$ ) a été déterminée par des analyses XPS, qui ont été réalisées sur un spectromètre ESCALAB 250i-XL équipé d'une source de rayons $X$ monochromatique $(\mathrm{Al} \mathrm{K \alpha})$. L'angle de collecte des photoélectrons est de $90^{\circ}$ par rapport à la surface de l'échantillon. Les énergies de liaison sont référencées par rapport au pic $\mathrm{Au}_{4 \mathrm{f} 7 / 2}$ situé à $83,8 \mathrm{eV}$. Les échantillons ont été analysés après avoir été rincés à l'eau bi distillée et séchés sous argon.

Les protéines adsorbées à la surface du substrat de $\mathrm{SnO}_{2}$ ont été analysées quantitativement et qualitativement. La désorption des protéines a été effectuée en chauffant les échantillons de $\mathrm{SnO}_{2}$ pendant 15 minutes à $90^{\circ} \mathrm{C}$ dans du tampon Tris pH 6,8 SDS $10 \%$. Le dosage biochimique des protéines solubilisées a ensuite été réalisé grâce à un kit commercialisé par Bio-Rad, basé sur une réaction colorimétrique selon la méthode de Lowry [17]. Les mesures ont été effectuées par spectrométrie en référence à une gamme étalon d'albumine (Pierce), les densités optiques étant mesurées à $750 \mathrm{~nm}$. Qualitativement, les protéines solubilisées sont analysées par électrophorèse en gel d'acrylamide selon la méthode de Laemmli [18]. Cette technique permet de séparer les protéines dont la vitesse de migration est proportionnelle à leur charge en SDS et à leur poids moléculaire. Les bandes protéiniques sont révélées par une coloration à l'argent qui permet de détecter des bandes contenant moins de $0,1 \mu \mathrm{g} \mathrm{cm}^{-2}$.

\section{Réponses électrochimique et électrogravimétrique de films $\mathrm{SnO}_{2}$ en présence de BSA}

La figure 1 montre l'effet de l'application d'un potentiel anodique sur l'adsorption de
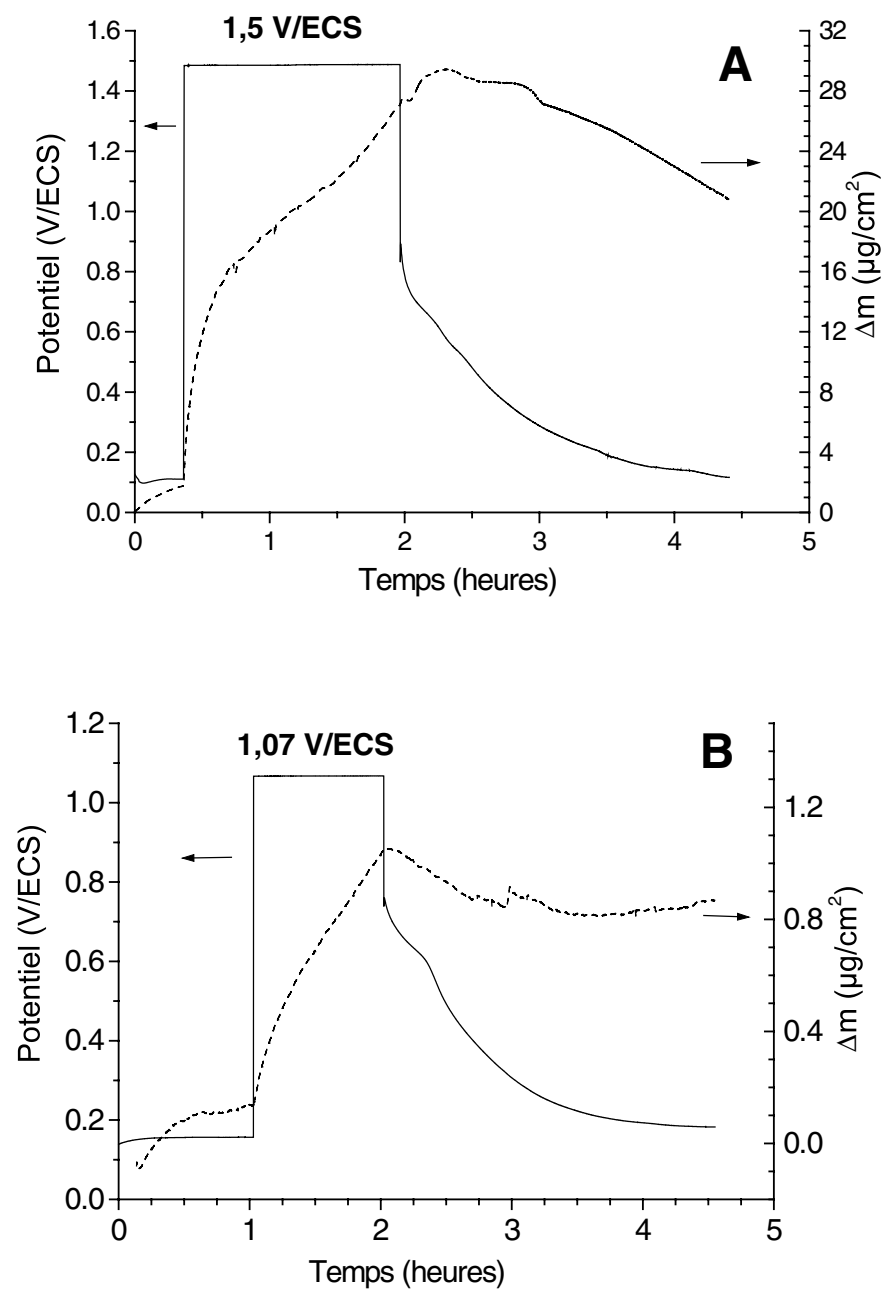

Fig. 1. Réponses chronoélectrogravimétrique et chronoampérométrique d'un film $\mathrm{SnO}_{2}$ en circuit ouvert, puis polarisé anodiquement à $+1,5 \mathrm{~V} / \mathrm{ECS}(\mathrm{A})$, ou à $+1,07 \mathrm{~V} / \mathrm{ECS}(\mathrm{B})$, puis à nouveau en circuit ouvert, dans du sérum de veau foetal + 0,5 $\mathrm{M} \mathrm{NaCl}$.

Fig. 1. Chronoelectrogravimetric and chronoamperometric curves of a $\mathrm{SnO}_{2}$ film initially at open circuit, then polarized at $+1.5 \mathrm{~V} / \mathrm{SCE}(\mathrm{A})$, or at $+1.07 \mathrm{~V} / \mathrm{SCE}(\boldsymbol{B})$, then again at open circuit, in foetal calf serum with $0.5 \mathrm{M}$ $\mathrm{NaCl}$. 

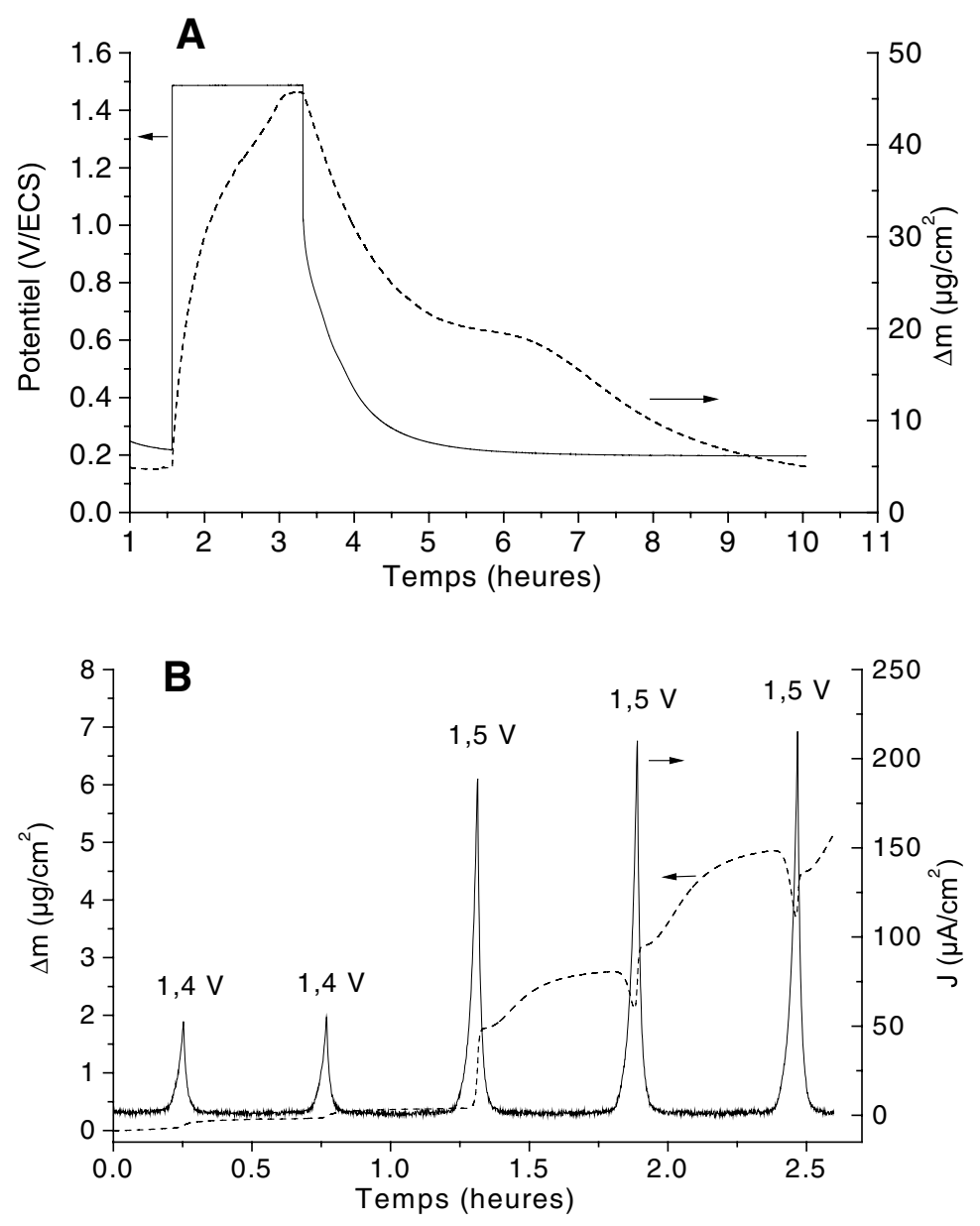

Fig. 2. (A) Réponses chronoélectrogravimétrique et chronoampérométrique d'un film $\mathrm{SnO}_{2}$ en circuit ouvert, puis maintenu à $+1,5 \mathrm{~V} /$ ECS, puis à nouveau en circuit ouvert, dans une solution de BSA à $0,2 \%+0,5 \mathrm{M} \mathrm{NaCl}$. (B) même expérience mais en cyclant le potentiel entre 0,5 et $+1,4 \mathrm{~V} / \mathrm{ECS}$, puis entre 0,5 et $1,5 \mathrm{~V} / \mathrm{ECS}$, à la vitesse de $1 \mathrm{mV} \mathrm{s}^{-1}$.

Fig. 2. (A) Chronoelectrogravimetric and chronoamperometric curves of a $\mathrm{SnO}_{2}$ film initially at open circuit, then held at $+1.5 \mathrm{~V} / \mathrm{SCE}$, then again at open circuit, in $0.5 \mathrm{M} \mathrm{NaCl}+0.2 \% \mathrm{BSA}$; $(B)$ same experiment but cycling the potential between 0.5 and $+1.4 \mathrm{~V} / \mathrm{SCE}$, then between 0.5 and $+1.5 \mathrm{~V} / \mathrm{SCE}$, at a scan rate of $1 \mathrm{mV} \mathrm{s}^{-1}$.

la BSA sur un film $\mathrm{SnO}_{2}$ au contact d'une solution $0,5 \mathrm{M} \mathrm{NaCl}$ contenant $5 \%$ de BSA, respectivement à $+1,5 \mathrm{~V} / \mathrm{ECS}$ (Fig. 1A) et $+1,07 \mathrm{~V} / \mathrm{ECS}$ (Fig. 1B). Initialement, au potentiel de repos, l'adsorption est faible. Elle augmente continûment en fonction du temps dès qu'un potentiel anodique est appliqué. Cette augmentation est d'autant plus importante que le potentiel est plus positif. Après coupure de la polarisation, on observe une légère décroissance de la masse adsorbée, avec simultanément une décroissance lente du potentiel de circuit ouvert vers une valeur limite voisine de $+0,2 \mathrm{~V} /$ ECS. La figure $2 \mathrm{~A}$ reproduit le même type d'expérience de chronoélectrogravimétrie que celles de la figure 1 , mise à part la concentration volumique en BSA qui est seulement de $0,2 \%$. L'adsorption sous polarisation anodique est toujours très conséquente, augmentant avec le temps de maintien de la polarisation. Par contre, après coupure de celle-ci, la décroissance de la masse est plus rapide en solution diluée de BSA $(0,2 \%)$ qu'en solution plus concentrée (5\%). La figure $2 \mathrm{~B}$ représente l'effet d'un cyclage du potentiel entre 0,5 et $+1,4 \mathrm{~V}$, puis entre 0,5 et $+1,5 \mathrm{~V}$, sur le courant anodique et sur la variation de la masse de l'électrode $\mathrm{SnO}_{2}$. Lorsque la limite anodique est $+1,4 \mathrm{~V}$, la masse de l'électrode évolue peu. L'extension de cette limite à $+1,5 \mathrm{~V}$ a un effet spectaculaire sur l'adsorption de la BSA. Il y a un gain de masse d'environ $1 \mu \mathrm{g} \mathrm{cm}^{-2}$ au passage vers $+1,5 \mathrm{~V}$, suivi par une augmentation pendant une partie du cycle, puis par un quasi plateau. La diminution observée au pied de la vague de courant pourrait correspondre à la corrosion du substrat entraînant une désorption partielle des protéines. Nos travaux antérieurs ont montré que ce mécanisme de corrosion a lieu dans un domaine étroit de potentiel (entre +1 et $+1,4 \mathrm{~V}$ ) et centré vers $+1,2 \mathrm{~V} / \mathrm{ECS}[5,19]$. À noter que cette séquence de corrosion est immédiatement suivie par un gain de masse de l'électrode, ce qui sous-entend que le mécanisme de corrosion de $\mathrm{SnO}_{2}$ en milieu chlorure en créant des états de surface chargés positivement jouerait un rôle actif dans le processus d'adsorption de la BSA globalement chargée négativement.

La figure 3A montre un enregistrement de chronoélectrogravimétrie en eau de mer naturelle (puisée dans la rade de Brest) dans laquelle on a ajouté de la BSA à la concentration de $0,3 \%$. Sous polarisation à $+1,5 \mathrm{~V}$, il y a une forte augmentation de la masse en fonction du temps, comme observé en milieu artificiel mais avec une vitesse de dépôt environ dix fois plus faible (voir Figs. 1A et 2A). La différence marquante est le quasi plateau observé sur une vingtaine d'heures après coupure de la polarisation, signifiant une très bonne stabilité de la couche de BSA adsorbée. La figure 3B illustre la réponse en courant et en masse de l'électrode $\mathrm{SnO}_{2}$ en eau de mer lors de cyclages du potentiel entre 0 et $+1,5 \mathrm{~V} / \mathrm{ECS}$. Le comportement est quelque peu différent de celui obtenu en milieu artificiel. La masse diminue légèrement pendant la durée d'un cycle. On ne voit pas de diminution de masse lorsque le potentiel est voisin de $+1,2 \mathrm{~V} /$ ECS. Comme en milieu artificiel, la masse de l'électrode augmente globalement au fur et à mesure 
des cycles, avec, dans le cas de l'eau de mer naturelle, un gain substantiel de $\approx 4 \mu \mathrm{g} \mathrm{cm}^{-2}$ à chaque passage à $+1,5 \mathrm{~V}$. On remarque qu'en eau de mer naturelle, le courant mesuré à chaque cycle à $+1,5 \mathrm{~V} / \mathrm{ECS}$ décroît significativement (environ $20 \%$ ) lorsque la masse adsorbée dépasse $\approx 10 \mu \mathrm{g} \mathrm{cm}^{-2}$.

La figure 4 illustre l'effet de la concentration volumique en chlorure dans le milieu électrolytique. Lorsque la concentration en chlorure passe de $0,15 \mathrm{M}$ (sérum physiologique) à $0,5 \mathrm{M}$ (proche de la concentration dans l'eau de mer), le courant anodique mesuré à $+1,5 \mathrm{~V}$ et en présence de BSA augmente (Fig. 4A). Cependant, l'augmentation n'est pas proportionnelle à la concentration volumique en chlorure, contrairement à ce qui a lieu en l'absence de BSA. La décroissance temporelle est caractéristique d'un contrôle diffusionnel du courant d'oxydation. Sur le temps d'observation (40 min), l'augmentation de masse est significative et d'autant plus grande que la concentration en ions chlorure est élevée (Fig. 4B).

L'examen des figures 1 à 4 permet d'estimer les vitesses de dépôt de la BSA sous polarisation à $+1,5 \mathrm{~V} /$ ECS. En milieu artificiel $\mathrm{NaCl}$, dans les toutes premières secondes, la vitesse atteint $40-50 \mathrm{ng} \mathrm{s}^{-1} \mathrm{~cm}^{-2}$. Elle décroît ensuite régulièrement pendant le premier quart d'heure, avec une vitesse moyenne autour de $20 \mathrm{ng} \mathrm{s}^{-1} \mathrm{~cm}^{-2}$. Au-delà, la vitesse se stabilise en fonction du temps, avec des valeurs entre 1 et $4 \mathrm{ng} \mathrm{s}^{-1} \mathrm{~cm}^{-2}$, la plus forte valeur correspondant à la plus faible concentration en BSA ( $0,2 \%)$. Le comportement en eau de mer est particulier : la vitesse moyenne de dépôt n'est que de $1,6 \mathrm{ng} \mathrm{s}^{-1} \mathrm{~cm}^{-2}$ aux temps courts, et $0,5 \mathrm{ng} \mathrm{s}^{-1} \mathrm{~cm}^{-2}$ aux temps longs. Enfin, l'effet du potentiel sur la vitesse de dépôt, évalué en milieu artificiel $\mathrm{NaCl} 0,5 \mathrm{M}$, est spectaculaire : $0,7 \mathrm{ng} \mathrm{s}^{-1} \mathrm{~cm}^{-2}$ aux temps courts, et $0,5 \mathrm{ng} \mathrm{s}^{-1} \mathrm{~cm}^{-2}$ aux temps longs à $+1,07 \mathrm{~V} / \mathrm{ECS}$, ces valeurs passant respectivement à 20 et $2 \mathrm{ng} \mathrm{s}^{-1} \mathrm{~cm}^{-2}$ pour un potentiel appliqué de $+1,5 \mathrm{~V} / \mathrm{ECS}$.

\section{Analyse par XPS de la composition chimique de la surface de $\mathrm{SnO}_{2}$}

La figure 5 montre les spectres XPS de films de $\mathrm{SnO}_{2}$ déposés sur des lames de verre, soit simplement immergés pendant 20 heures, soit polarisés à $+1,5 \mathrm{~V}$ pendant 20 heures également, dans du sérum $0,15 \mathrm{M} \mathrm{NaCl}$ avec de la BSA à une concentration de $3 \%$. Les
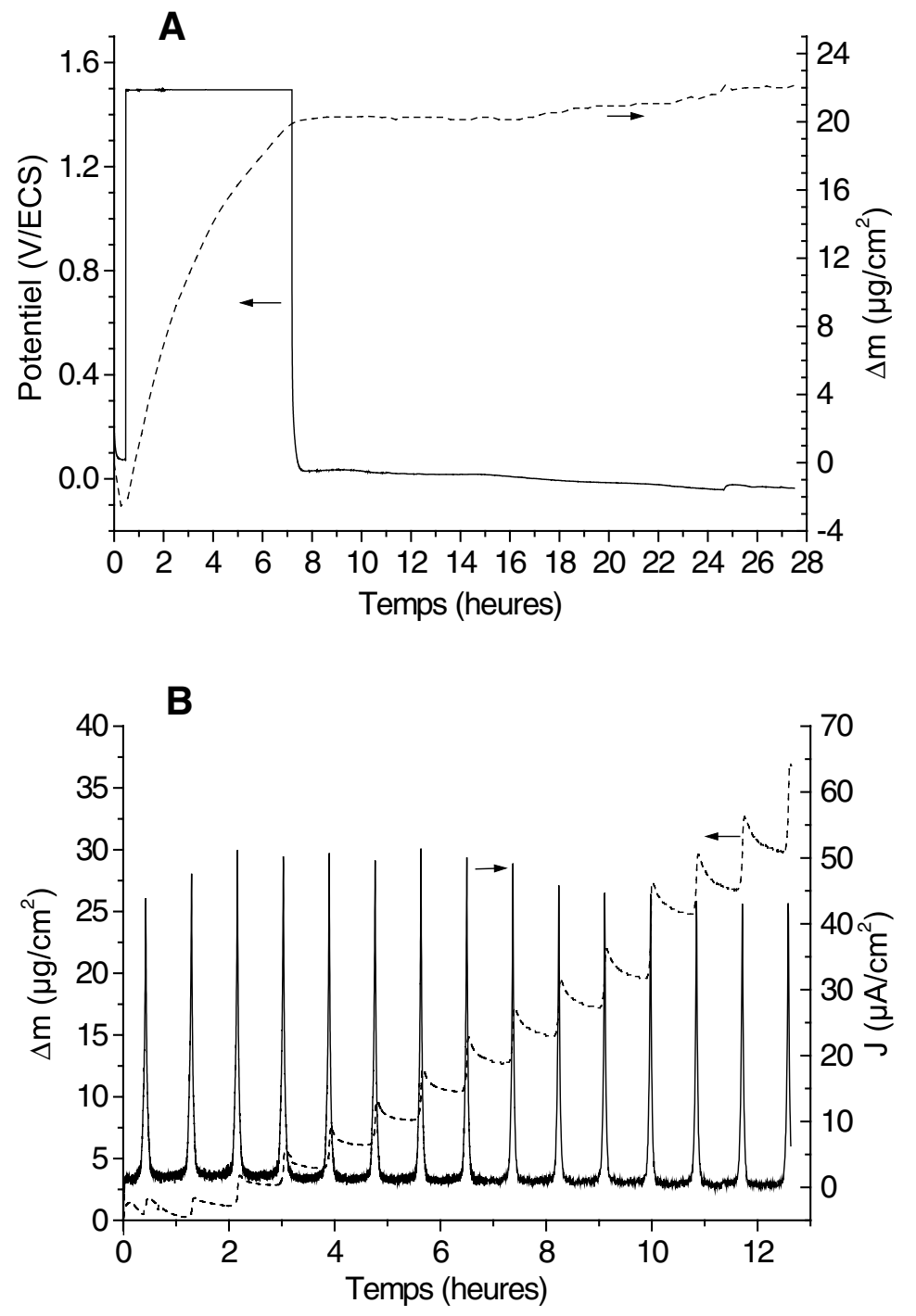

Fig. 3. (A) Réponses chronoélectrogravimétrique et chronoampérométrique d'un film $\mathrm{SnO}_{2}$ en circuit ouvert, puis maintenu à $+1,5 \mathrm{~V} /$ ECS, puis à nouveau en circuit ouvert, dans l'eau de mer naturelle + BSA à $0,3 \%$. (B) même expérience mais en cyclant le potentiel entre 0 et $+1,5 \mathrm{~V} / \mathrm{ECS}$, à la vitesse de $1 \mathrm{mV} \mathrm{s}^{\mathbf{- 1}}$.

Fig. 3. (A) Chronoelectrogravimetric and chronoamperometric curves of a $\mathrm{SnO}_{2}$ film initially at open circuit, then held at $+1.5 \mathrm{~V} / \mathrm{SCE}$, then again at open circuit, in natural seawater $+0.3 \%$ BSA; $(B)$ same experiment but cycling the potential between 0 and $+1.5 \mathrm{~V} / \mathrm{SCE}$ at a scan rate of $1 \mathrm{mV} \mathrm{s}^{-1}$.

spectres de ces deux échantillons sont à comparer à celui d'un échantillon témoin, tel que fabriqué. Après une simple immersion, on voit apparaître la contribution des photopics $\mathrm{N} 1 \mathrm{~s}$ et $\mathrm{C} 1 \mathrm{~s}$, caractéristiques de la matière organique, mais la signature du substrat $\mathrm{SnO}_{2}$ est toujours nettement présente. Par contre, après 20 heures sous polarisation, les pics N1s et C1s sont beaucoup plus intenses et la contribution du substrat a maintenant totalement disparu. On détecte également du soufre, dû aux acides aminés tels que la cystéine et la méthionine. Ainsi, en circuit ouvert, il y a une faible adsorption de la BSA, 


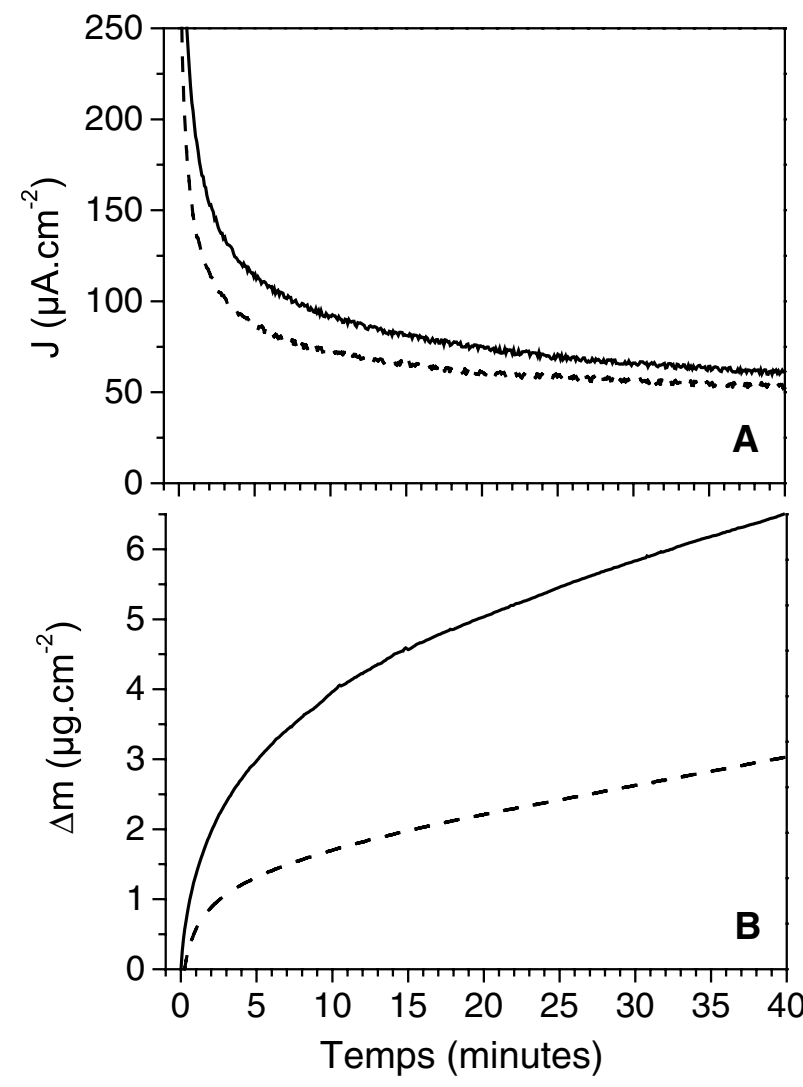

Fig. 4. Effet de la concentration volumique en $\mathrm{NaCl}$ sur les réponses chronoampérométrique $(\mathrm{A})$ et chronoélectrogravimétrique (B) d'un film $\mathrm{SnO}_{2}$ polarisé à $+1,5 \mathrm{~V} /$ ECS dans du SVF (---), en présence de $\mathrm{NaCl} \mathrm{0,5} \mathrm{M} \mathrm{(-).}$

Fig. 4. Dependence of the chronoamperometric $(A)$ and chronoelectrogravimetric (B) curves of a $\mathrm{SnO}_{2}$ film on the bulk $\mathrm{NaCl}$ concentration, when polarized at $+1.5 \mathrm{~V} / \mathrm{SCE}$ in foetal calf serum (--), in the presence of $0.5 \mathrm{M} \mathrm{NaCl}$ (-).

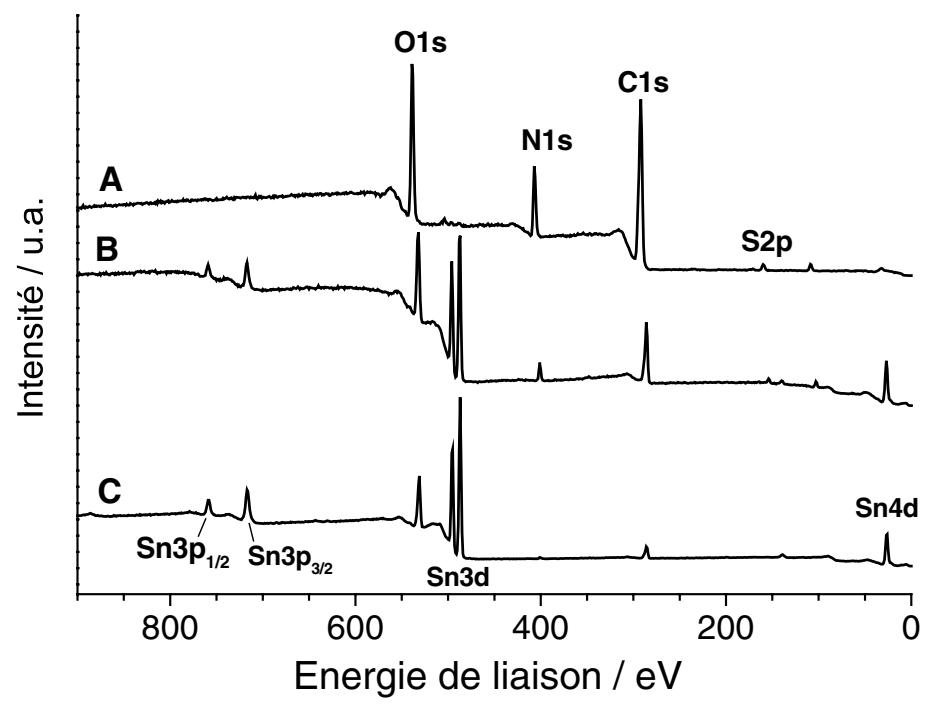

Fig. 5. Spectres XPS de films $\mathrm{SnO}_{2}$, (A) après immersion sous polarisation à $+1,5 \mathrm{~V} / \mathrm{ECS}$ pendant 20 heures, dans une solution $0,15 \mathrm{M} \mathrm{NaCl}+\mathrm{BSA}$ à $3 \%$, (B) après immersion en circuit ouvert pendant 20 heures, $(\mathrm{C})$ tel que fabriqué.

Fig. 5. XPS spectra of $\mathrm{SnO}_{2}$ films, (A) polarized at $1.5 \mathrm{~V} / \mathrm{SCE}$ for 20 hours in $0.15 \mathrm{M} \mathrm{NaCl}+3 \% \mathrm{BSA},(B)$ immersed at open circuit potential for 20 hours, $(C)$ as deposited. qui ne suffit pas à masquer la réponse du substrat. Après polarisation anodique à $+1,5 \mathrm{~V}$, la couche de BSA adsorbée est plus importante, son épaisseur est supérieure à 5$10 \mathrm{~nm}$ puisque le substrat $\mathrm{SnO}_{2}$ n'est plus détecté.

La figure 6 montre le spectre XPS d'un film de $\mathrm{SnO}_{2}$ ayant été polarisé deux heures à $+1,5 \mathrm{~V}$ dans une solution $0,5 \mathrm{M} \mathrm{NaCl}$ renfermant $0,1 \%$ de BSA. Ici encore, la contribution du film de $\mathrm{SnO}_{2}$ n'est plus visible. Par contre, outre les photopics dus à l'azote, au carbone, à l'oxygène et au soufre (caractéristiques de la BSA), du chlore est également détecté. Nous avons vérifié qu'il n'y avait pas de signal correspondant au sodium, ce qui aurait pu indiquer un rinçage insuffisant. Le chlore est donc lié à la matière organique.

\section{Analyse des protéines adsorbées}

Des échantillons de $\mathrm{SnO}_{2}$ ont été immergés dans du SVF qui contient majoritairement de l'albumine en quantité estimée à 3-4\%. La concentration en $\mathrm{NaCl}$ a été, selon le cas, ajustée à $0,5 \mathrm{M}$ par addition de $\mathrm{NaCl}$, pour se rapprocher de la concentration saline de l'eau de mer et les échantillons laissés en circuit ouvert ou polarisés à $+1,5 \mathrm{~V} / \mathrm{ECS}$ pendant 1 heure à $37^{\circ} \mathrm{C}$. Les résultats des dosages protéiniques sont rapportés dans le tableau 1. On constate que dans nos conditions expérimentales, les quantités de protéines adsorbées sont faibles. Leur pourcentage est inférieur à $0,1 \%$ des protéines sériques totales. La polarisation a pour effet d'augmenter la quantité de protéine adsorbée, effet exacerbé par l'addition de $\mathrm{NaCl}$ à $0,5 \mathrm{M}$. Ces résultats sont en accord avec ceux obtenus par électrogravimétrie.

La figure 7 montre les résultats des analyses électrophorétiques. Une bande majoritaire apparaît dans la zone de migration de l'albumine bovine 66-67 kD, quel que soit le traitement subi par les échantillons. Aucune bande n'apparaît dans la zone des protéines supérieures à $100 \mathrm{kD}$. On en déduit que la fibronectine, protéine de haut poids moléculaire, fortement adhésive et présente en faible quantité dans le sérum n'est pas détectable dans ces conditions ou en trop faible quantité par rapport à l'albumine. Quantitativement, la figure 7 met en évidence une augmentation des protéines adsorbées en condition de polarisation (pistes 4 et 5) par rapport aux conditions de non polarisation (pistes 2 et 3). L'augmentation de l'adsorption en présence de $\mathrm{NaCl}$ 0,5 $\mathrm{M}$ est révélée 


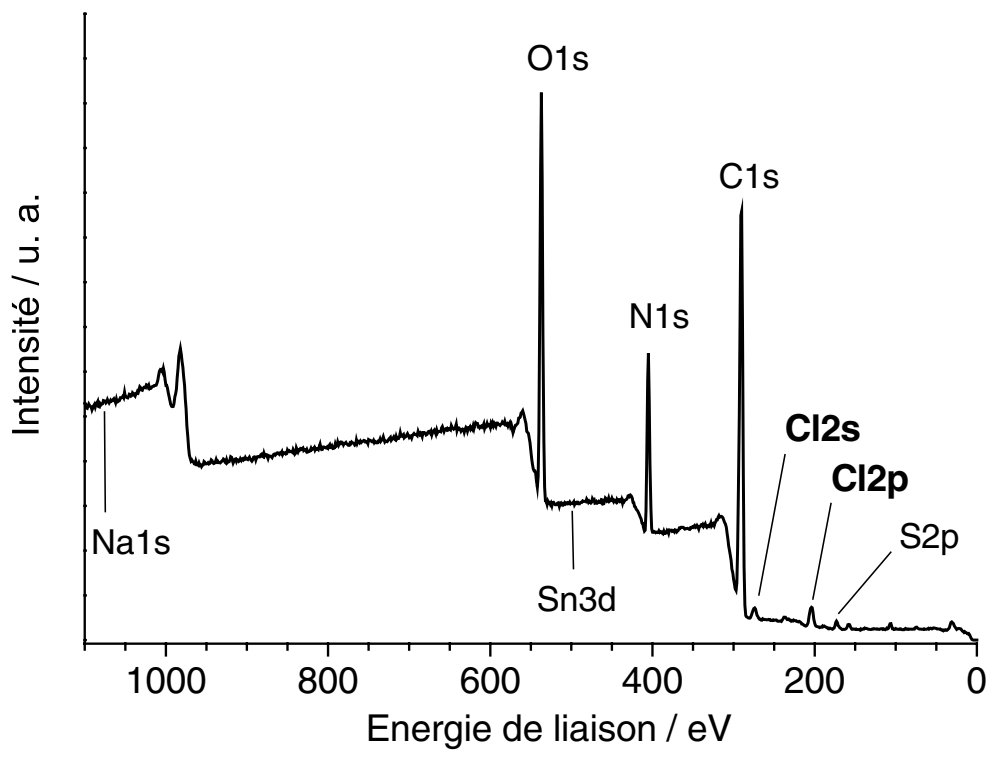

Fig. 6. Spectre XPS d'un film $\mathrm{SnO}_{2}$ après polarisation à $+1,5 \mathrm{~V} / \mathrm{ECS}$ pendant 2 heures dans une solution $0,5 \mathrm{M} \mathrm{NaCl}+\mathrm{BSA}$ à $\mathbf{0 , 1} \%$.

Fig. 6. XPS spectrum of a $\mathrm{SnO}_{2}$ film after being polarized at $+1.5 \mathrm{~V} / \mathrm{SCE}$ for 2 hours in $0.5 \mathrm{M} \mathrm{NaCl}+0.1 \% \mathrm{BSA}$.
Tableau 1. Adsorption de BSA sur des échantillons de $\mathrm{SnO}_{2}$, soit polarisés à $+1,5 \mathrm{~V} /$ ECS pendant 1 heure, soit simplement immergés pendant 1 heure également.

Table 1. Adsorption of BSA on $\mathrm{SnO}_{2}$ samples, either polarized at $+1.5 \mathrm{~V} / \mathrm{SCE}$ for 1 hour, or simply immersed for the same time.

\begin{tabular}{|c|c|c|}
\hline $\begin{array}{c}\text { Conditions } \\
\text { expérimentales }\end{array}$ & $\mathrm{NaCl}$ & $\begin{array}{c}\text { BSA adsorbée } \\
\mu \mathrm{g} \mathrm{cm}^{-2}\end{array}$ \\
\hline Polarisation à +1,5V/ECS & $0,15 \mathrm{M}$ & 5,6 \\
Simple immersion & $0,15 \mathrm{M}$ & 1,9 \\
\hline Polarisation à +1,5 V/ECS & $0,5 \mathrm{M}$ & 6,9 \\
Simple immersion & $0,5 \mathrm{M}$ & 2,2 \\
\hline
\end{tabular}

par la comparaison des pistes 4 et 5, les bandes étant plus prononcées en présence de $\mathrm{NaCl}$ 0,5 M (piste 5).

La durée de polarisation augmente la quantité de protéines adsorbées comme le montre la figure 8 . Ces expériences ont été réalisées en présence d'une solution à $0,1 \%$ de $\mathrm{BSA}$ et $0,5 \mathrm{M} \mathrm{NaCl}$. L'électrophorèse révèle une seule bande à $66 \mathrm{kD}$ dont l'intensité augmente avec la durée de polarisation, des volumes identiques d'éluats ayant été déposés sur les pistes 2 et 3 . Des essais de polarisation prolongée jusqu'à 48 heures ont montré un accroissement de la quantité d'albumine adsorbée en fonction du temps. L'intensité du marquage de la bande 66 kD est nettement plus accentuée bien que l'éluat ait été 6 fois plus dilué que les précédents. $\mathrm{D}^{\prime}$ autre part, la présence d'une seule bande de migration témoigne de l'absence de fragmentation de l'albumine adsorbée lors de la polarisation anodique.

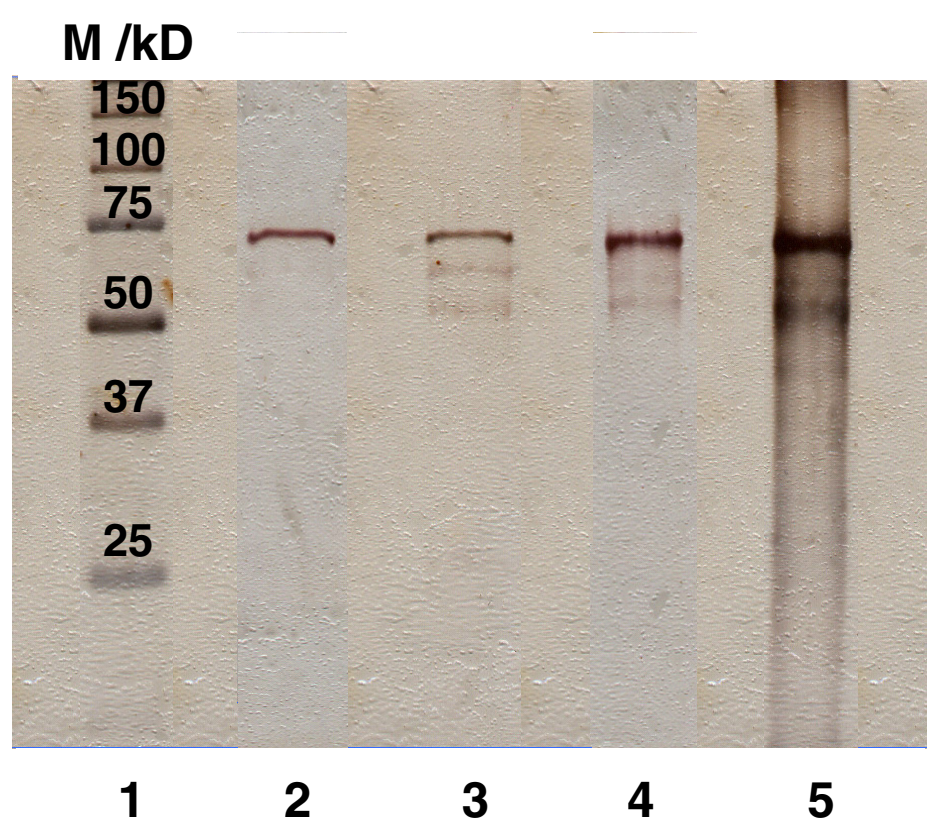

Fig. 7. Analyse électrophorétique des protéines sériques adsorbées sur des échantillons de $\mathrm{SnO}_{2}$ incubé 1 heure dans du SVF (2), incubé 1 heure dans du SVF additionné de $\mathrm{NaCl} 10,5 \mathrm{M}(3)$, polarisé 1 h30 dans du SVF à +1,5 V/ECS (4), identique à (4) mais SVF additionné de $\mathrm{NaCl}$ 0,5 M (5), marqueurs moléculaires (1).

Fig. 7. Electrophoretic analysis of seric proteins adsorbed on $\mathrm{SnO}_{2}$ samples incubated for 1 hour in foetal calf serum (2), with $0.5 \mathrm{M} \mathrm{NaCl}$ added (3), polarized at $+1.5 \mathrm{~V} / \mathrm{SCE}$ for 1 hour (4), as (4) but $0.5 \mathrm{M} \mathrm{NaCl}$ added (5); marker (1).

\section{Discussion et conclusion}

Les études électrochimiques et biochimiques que nous avons réalisées montrent conjointement que la protéine BSA s'adsorbe sur $\mathrm{SnO}_{2}$. Cette adsorption est faible en circuit ouvert, comparable à ce qui est observé dans la littérature $[1,2,8,9]$. En revanche, 


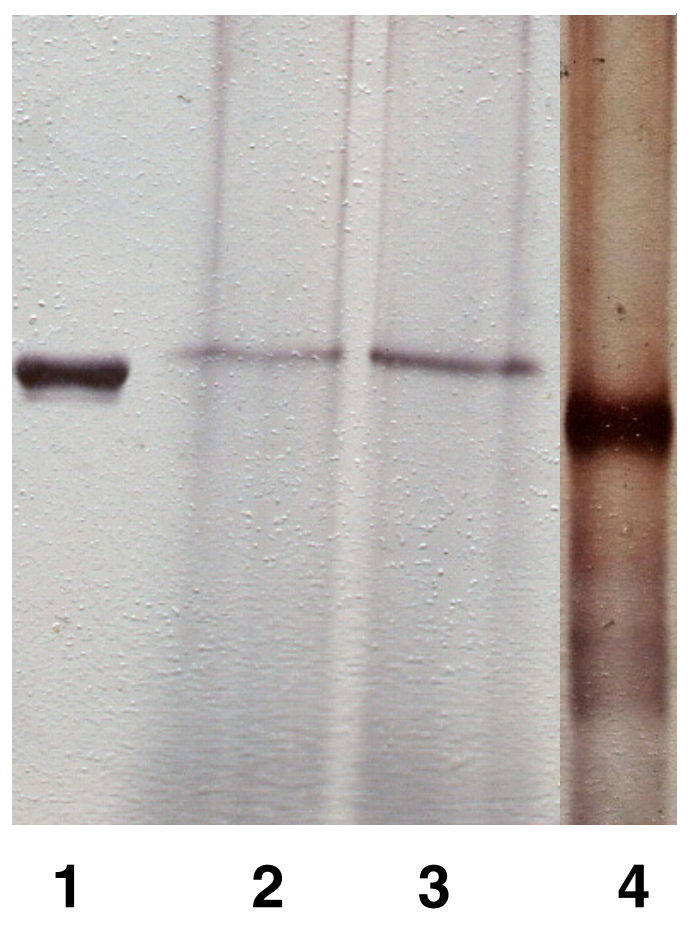

Fig. 8. Analyse électrophorétique de BSA adsorbée sur des échantillons de $\mathrm{SnO}_{2}$ polarisés à $+1,5 \mathrm{~V} / \mathrm{ECS}$ en présence de $\mathrm{NaCl} 0,5 \mathrm{M}$ pendant $30 \mathrm{~min}$. (2), 2 heures (3) 48 heures (4), marqueur BSA (1).

Fig. 8. Electrophoretic analysis of BSA adsorbed on $\mathrm{SnO}_{2}$ samples polarized at $+1.5 \mathrm{~V} / \mathrm{SCE}$ in the presence of $0.5 \mathrm{M} \mathrm{NaCl}$ for 0.5 hour (2), for 2 hours (3), for 48 hours (4), marker BSA (1).

en présence d'ions chlorure, elle devient très conséquente dès que le potentiel atteint $+1,5 \mathrm{~V} /$ ECS. À ce potentiel, d'une part, en l'absence de BSA, il y a production d'acide hypochloreux proportionnellement au courant faradique, d'autre part, il y a formation d'une concentration d'états de surface chargés positivement, concentration stationnaire pour un courant donné. Ceux-ci résultent de l'interaction avec la surface de $\mathrm{SnO}_{2}$ de radicaux $\mathrm{OH}^{\circ}$ et $\mathrm{Cl}^{\circ}$, qui sont des espèces intermédiaires dans les réactions d'oxydation de $\mathrm{OH}^{-}$et $\mathrm{Cl}^{-}$. Dans le cas de la BSA, chargée négativement aux $\mathrm{pH}$ supérieurs à $\approx 5$, la charge de surface positive peut initier l'adhésion de la BSA par interactions électrostatiques. Toutefois, cet argument ne semble pas suffisant pour expliquer la croissance d'une couche plus épaisse qu'une monocouche. En effet, la quantité théorique de BSA pour former une monocouche est de $1,8 \times 10^{12}$ molécules par $\mathrm{cm}^{2}$, soit $0,2 \mu \mathrm{g} \mathrm{cm}^{-2}$, pour une adsorption parallèle à la surface, et de $6,25 \times 10^{12}$ molécules par $\mathrm{cm}^{2}$, soit $0,7 \mu \mathrm{g} \mathrm{cm}^{-2}$, pour une adsorption perpendiculaire à la surface. Sous polarisation anodique de $+1,5 \mathrm{~V} / \mathrm{ECS}$, nous avons trouvé qu'à température ambiante, l'adsorption de la BSA correspondait à un gain de masse de $\approx 30 \mu \mathrm{g} \mathrm{cm}^{-2}$, soit environ $3 \times 10^{14}$ molécules de BSA par $\mathrm{cm}^{2}$. Dans nos conditions de polarisation à $+1,5 \mathrm{~V} / \mathrm{ECS}$, la concentration surfacique en BSA est donc très largement supérieure à une monocouche, quel que soit le mode d'organisation de la BSA par rapport à la surface de $\mathrm{SnO}_{2}$. Compte tenu des vitesses de dépôt estimées, la première monocouche est complétée en quelques secondes en milieu artificiel, et en quelques minutes en eau de mer naturelle. Les tests biochimiques effectués à $37^{\circ} \mathrm{C}$ montrent une adsorption encore plus conséquente. À noter qu'à un potentiel moins anodique (+1,07 V/ECS), l'adsorption de la BSA a lieu à une vitesse environ dix fois plus faible qu'au potentiel de $+1,5 \mathrm{~V} / \mathrm{ECS}$, avec, au bout d'une heure, une quantité adsorbée de l'ordre de la monocouche (Fig. 1B).

Par ailleurs, il est clair que la croissance de la couche organique s'effectue alors que $\mathrm{HClO}$ est produit électrochimiquement. On doit donc prendre en compte l'interaction de $\mathrm{HClO}$ avec les protéines. Il a été montré dans la littérature que $\mathrm{HClO}$ interagissait préférentiellement avec les chaînes latérales contenant des fonctions amine primaire (i.e. lysine), créant des liaisons $\mathrm{N}-\mathrm{Cl}$ correspondant à la formation de groupements chloramine portés par le squelette de la protéine, eux-mêmes pouvant évoluer vers la formation de radicaux centrés sur un atome d'azote [12-14]. Pour l'instant, nous ne pouvons conclure sur l'effet de l'interaction BSA/HClO sur la croissance de la couche organique. Le seul point sur lequel nous pouvons conclure est celui de l'incorporation de chlore dans la matière organique adhérant à la surface de $\mathrm{SnO}_{2}$ après polarisation en milieu chloré. Il faut remarquer que le signal du chlore a été détecté dans le cas du dépôt formé à partir d'une solution peu concentrée, à $0,1 \%$ en BSA pour un temps de polarisation à $+1,5 \mathrm{~V} /$ ECS de 2 heures (Fig. 6).

Par contre, pour une concentration en BSA de $3 \%$ et un temps de maintien de 20 heures (Fig. 5C), aucun signal du chlore n'a pu être détecté. Ce résultat suggère que le rapport entre la concentration en BSA présente en solution et la quantité de $\mathrm{HClO}$ formée à l'électrode est un paramètre important qui influe sur l'incorporation de chlore dans la couche organique : une valeur élevée du rapport $[\mathrm{HClO}] /[\mathrm{BSA}]$ favoriserait le piégeage du chlore dans la couche. D'autre part, nous avons observé, dans une expérience indépendante sur le système $\mathrm{NaCl} 0,5 \mathrm{M}+\mathrm{BSA}$ 
à $3 \%$, le début d'une perte de masse sous polarisation après un temps de maintien d'environ 7 heures. Pour une concentration de BSA de $3 \%$, la masse déposée " critique » serait de l'ordre de $30 \mathrm{\mu g} \mathrm{cm}^{-2}$; elle serait plus élevée en solution de BSA à $0,2 \%$, atteignant $45 \mathrm{\mu g} \mathrm{cm}^{-2}$ (Fig. 2A). Les dépôts effectués en milieu BSA dilué auraient donc une meilleure stabilité que ceux formés en solution concentrée en BSA, bien que les vitesses de dépôt soient peu différentes. Ce constat est à mettre en regard du comportement très particulier observé en eau de mer naturelle. La vitesse de dépôt est faible mais la stabilité de l'adsorbat est remarquable. Une telle différence de comportement pourrait être liée à la différence d'homogénéité des couches déposées.

Cet article montre clairement qu'il existe une corrélation entre le dégagement de $\mathrm{HClO}$ et l'adsorption de la BSA à la surface de $\mathrm{SnO}_{2}$. Toutefois, le mécanisme réactionnel reste à expliciter. Par ailleurs, il est important de signaler que les analyses XPS ont été effectuées sur des échantillons sortis $\mathrm{du}$ milieu liquide, rincés abondamment, séchés et stockés plusieurs jours. Ceci signifie que le film organique est bien adhérent et que la modification induite par le chlore est stable dans le temps. Si l'on admet que la présence de chlore reflète la formation de groupements chloramine, on peut penser avoir recouvert la surface de $\mathrm{SnO}_{2}$ par un film organique ayant des propriétés biocides. Une des étapes ultérieures de ce travail consistera à apprécier ce caractère biocide par des tests de colonisation bactérienne.

\section{Références}

[1] C. Poleunis, C. Rubio, C. Compère, P. Bertrand, Appl. Surf. Sci. 203-204 (2003) 693
[2] A.N. Asanov, L.J. DeLucas, P.B. Oldham, W.W. Wilson, J. Coll. Interf. Sci. 191 (1997) 222

[3] H. Cachet, S. Haskouri, G. Folcher, B. Tribollet, D. Festy, Mat. Tech. 7/8 (2004) 9

[4] H. Cachet, D. Festy, G. Folcher, F. Mazeas, B. Tribollet, Mat. Tech. 7/8 (2002) 37

[5] H. Cachet, F. Zenia, M. Froment, J. ElectroChem. Soc. 146 (1999) 977

[6] Stacie E. Dunkle, "Romarchite and other corrosion phases on metal artefacts from the Queen Anne's Revenge (1718)". Master of Science in Geological Sciences, April 2002, Virginia Polytechnic Institute and State University, Blacksburg, Virginia (USA)

[7] H. Cachet, S. Haskouri, C. DebiemmeChouvy, D. Festy, Antifouling properties of surface modified tin oxide films immersed at rest potential in chloride media after an anodic polarisation step, Proceedings of Eurocorr 2004, EFC event n²66, Workshop "Biofilms and materials in natural environments", Nice September 2004

[8] Z.G. Peng, K. Hidajat, M.S. Uddin, J. Coll. Interf. Sci. 271 (2004) 277

[9] Y. Zhang, Y. Fung, H. Sun, D. Zhu, S. Yao, Sensors and Actuators B 108 (2005) 933

[10] W.K. Lee, J.S. Ko, H.M. Kim, J. Coll. Interf. Sci. 246 (2002) 70

[11] K.Y. Chun, P. Stroeve, Langmuir 18 (2002) 4653

[12] C.L. Hawkins, M.J. Davies, Biochem. J. 332 (1998) 617

[13] C.L. Hawkins, M.J. Davies, Biochem. J. 340 (1999) 539

[14] C.L. Hawkins, M.D. Rees, M.J. Davies, FEBS Lett. 510 (2002) 41

[15] SDS-PAGE : Sodium Dodecylsulfate PolyAcrylamide Gel Electrophoresis

[16] K. Bizet, C. Gabrielli, H. Perrot, Appl. Biochem. Biotech. 89 (2000) 139

[17] O.H. Lowry, N.J. Rosenbrough, A.L. Farr, R.J. Randall, J. Biol. Chem. 193 (1951) 265

[18] U.K. Laemmli, Nature 227 (1970) 680

[19] H. Cachet, M. Froment, F. Zenia, J. ElectroChem. Soc. 143 (1996) 442 